\title{
Trap Coverage: Allowing Coverage Holes of Bounded Diameter in Wireless Sensor Networks
}

\author{
Paul Balister ${ }^{\S} \quad$ Zizhan Zheng ${ }^{\dagger}$ \\ $\S$ University of Memphis \\ \{pbalistr,santosh.kumar\}@memphis.edu
}

\author{
Santosh Kumar ${ }^{\S}$ \\ Prasun Sinha ${ }^{\dagger}$ \\ $\dagger$ The Ohio State University \\ \{zhengz,prasun\}@cse.ohio-state.edu
}

\begin{abstract}
Tracking of movements such as that of people, animals, vehicles, or of phenomena such as fire, can be achieved by deploying a wireless sensor network. So far only prototype systems have been deployed and hence the issue of scale has not become critical. Real-life deployments, however, will be at large scale and achieving this scale will become prohibitively expensive if we require every point in the region to be covered (i.e., full coverage), as has been the case in prototype deployments.

In this paper we therefore propose a new model of coverage, called Trap Coverage, that scales well with large deployment regions. A sensor network providing Trap Coverage guarantees that any moving object or phenomena can move at most a (known) displacement before it is guaranteed to be detected by the network, for any trajectory and speed. Applications aside, trap coverage generalizes the de-facto model of full coverage by allowing holes of a given maximum diameter $(d)$. From a probabilistic analysis perspective, the trap coverage model explains the continuum between percolation (when coverage holes become finite) and full coverage (when coverage holes cease to exist).

We take first steps toward establishing a strong foundation for this new model of coverage. We derive reliable, explicit estimates for the density needed to achieve trap coverage with a given diameter when sensors are deployed randomly. We show by simulation that our analytical predictions of density are quite accurate even for small networks. Next, we investigate optimal deterministic patterns for deployment. We show that for $d \leq 0.5552 r$, where $r$ is the sensing range, the optimal deployment pattern is a triangular grid and for large $d / r$, the subdivided hexagonal grid is within $10 \%$ of optimal. Proving the exact optimal pattern appears to be an extremely difficult problem, related to several open problems in optimal plane packing. Finally, we propose polynomial-time algorithms to determine the level of trap coverage achieved once sensors are deployed on the ground.
\end{abstract}

\section{INTRODUCTION}

Several promising applications of wireless sensor networks with a high potential to impact human society involve detection and tracking of movements. Movements may be of persons, animals, and vehicles, or of phenomena such as fire. Examples include tracking of thieves fleeing with stolen objects in a city, tracking of intruders crossing a secure perimeter, tracking of enemy movements in a battlefield, tracking of animals in forests, tracking the spread of forest fire, and monitoring the spread of crop disease.

So far only prototype systems have been deployed and hence the issue of scale has not become critical. Real-life deployments, however, will be at large scale, and achieving this scale will become prohibitively expensive if we require every point in the region to be covered (i.e., full coverage or blanket coverage [19]), as has been the case in prototype deployments [14], [17], [22]. The requirement of full coverage will soon become a bottleneck as we begin to see real-life deployments.

In this paper, we therefore propose a new model of coverage, called Trap Coverage, that scales well with large deployment regions. We define a Coverage Hole in a target region of deployment $\mathcal{A}$ to be a connected component ${ }^{1}$ of the set of uncovered points of $\mathcal{A}$. A sensor network is said to provide Trap Coverage with diameter $d$ to $\mathcal{A}$ if the diameter of any Coverage Hole in $\mathcal{A}$ is at most $d$. For every deployment that provides trap coverage with diameter of $d$, the sensor network guarantees that every moving object or phenomena of interest will surely be detected for every displacement $d$ that it travels in $\mathcal{A}$. At any instant, we can either pin point the location of a moving object precisely, or can point to a coverage hole of diameter at most $d$ in which it is trapped.

With this model, the density of sensors can be adjusted to meet the desired quality of tracking while economizing on the number of sensors needed. Large scale sensor deployments for tracking thus become economically feasible with this new model of coverage. Figure 1 shows an example deployment region where the size of the largest uncovered region is $d$.

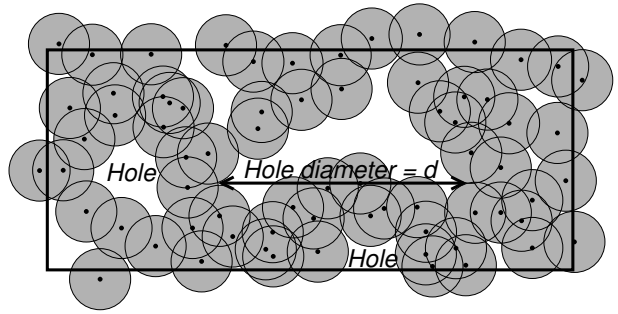

Fig. 1. In this deployment, $d$ is the diameter of the largest hole. Notice that although the diameter line intersects a covered section, it still represents the largest displacement that a moving object can travel within the target region without being detected.

Trap Coverage Generalizes Full Coverage: If the value of $d$ is set to 0 , then trap coverage is equivalent to full coverage. By relaxing the requirement of having every point covered, trap coverage generalizes the model of full coverage.

Traditionally, the fraction of target region that is covered has been used as an indicator of the quality of coverage [14], [26]. Notice that even if a large fraction of region is covered, the diameter of the largest hole may be arbitrarily large. Therefore,

\footnotetext{
${ }^{1}$ Here connected refers to the connectivity of a set of points in the real plane that comprise the target region.
} 
trap coverage may better indicate the Quality of Full Coverage as it provides a deterministic guarantee in the worst case.

\section{Key CONTRIBUTIONS AND RoAdMAP}

In addition to introducing a new model that generalizes the traditional full coverage model, we make several contributions in this paper, some of which may be of independent interest.

First, we derive a reliable estimate of the density (similar as in [3]) needed to achieve trap coverage with a desired diameter $d$ when sensors are deployed randomly. Roughly speaking, the critical density condition is of the form

$$
\lambda\left(2 r d+\pi r^{2}\right) \approx \log n,
$$

where $\lambda$ is the expected density of sensors per unit area, $r$ is the sensing range, and $n=\lambda|\mathcal{A}|$ is the expected total number of sensors in the target region $\mathcal{A}$. In other words, we expect that having, on average, $\log n$ sensors in the $r$ neighborhood of a thin long hole of diameter $d$ will suffice for achieving trap coverage with a diameter of $d$. We also show how our estimate for the density can be adapted to a non-disk model of sensing region, by using ellipses of random orientation as an example. (Section IV)

Second, the model of trap coverage explains the gap that has long existed between the percolation threshold (when holes become finite and isolated) and the critical density for achieving full coverage (when holes cease to exist). Looking at (1), we can observe that if $r$ is constant w.r.t. $n$, which is the case for percolation to occur, $d$ is of the order of $\log n$, matching the known behavior that for fixed $\lambda r^{2}$ above the percolation threshold, the maximum hole diameter is on average of order $\log n$. On the other hand, if $d$ is a constant, and 0 in particular, then $\lambda r^{2}$ is of the order of $\frac{1}{\pi} \log n$, matching the known behavior for achieving full coverage [19]. Thus, the trap coverage model not only generalizes the model of full coverage, it also helps explain the probabilistic behavior of coverage between the percolation threshold and critical density for full coverage. (See Figure 14 for an illustration.)

Third, for deterministic deployments, we show that for $d \leq 0.5552 r$, the optimal deployment pattern is a triangular grid. We conjecture that this is in fact optimal for all $d \lesssim$ $1.1292 r$, a square grid is optimal for $1.1292 r \lesssim d \lesssim 2.0798 r$, and a hexagonal grid is optimal for larger $d$, where we may subdivide the edges of the hexagonal grid a number of times if $d$ is large (see Figure 6). For all $d$, we give good bounds on the optimal deployment density, showing in particular that the subdivided hexagonal grid is within $10 \%$ of optimal for large $d / r$. However, proving the exact optimal pattern appears to be an extremely difficult problem, related to several open problems in optimal plane packing. (Section V)

Once sensors have been deployed on the ground (either randomly or deterministically), it may be necessary to determine the level of trap coverage that they provide, since some may fail at or after the deployment for unforeseen reasons. Our third contribution, therefore, is polynomial time algorithms to determine the level of trap coverage that an arbitrary deployed sensor network provides. Our algorithms not only works for non-convex models of sensing regions, but also when sensing regions are uncertain (e.g., probabilistic sensing models). Further, they take into consideration the complications that may arise due to the boundary of the deployment region (see Figure 10 for an example). (Section VI)

\section{RELATED WORK}

Most work on probabilistic density estimates for coverage assume the full coverage model [19], [25], [31]. As we show in Section IV, the naïve approach of increasing the sensing range by $d$ and then deriving the conditions for full coverage will lead to overdeployment, no matter how small the value of $d>0$ is. For larger $d$, overdeployment will be orders of magnitude more than needed in our estimates.

Work on full coverage that does consider holes focuses on the fraction of region that is (un)covered, see [25], [31]. They attempt to asymptotically minimize the area of vacant region and do not provide any simple expression for the density needed in a random deployment to achieve a desired fraction of uncovered region. Even if there existed such an expression, it could not be used to readily derive an estimate of density needed for bounding the diameter of coverage holes. This is because holes of large diameter tend to be long and thin, and their area is not typically large (even close to zero).

Perhaps, the work closest to trap coverage are [8], [11] that allow holes for surveillance applications. Here the quality of surveillance metric is based on the distance that a moving target, starting at a random location, moving in a random direction can travel in a straight line before it is detected by a sensor. In [8], distance to detection by a giant connected component is also studied. There are several issues with such a metric. For one, they do not provide any worst case guarantee on how far a target can move before being detected, unlike trap coverage. For example, if the density chosen is just large enough that a giant component exists almost surely, as in [8], the hole diameters are not bounded by any constant; they grow as a function of $\log n$ where $n$ is the number of sensors deployed. Further, even though the average distance may be bounded, even close to zero, the worst case distance could be arbitrarily large (as show in Figure 2). As shown in a typical deployment (Figure 4), holes that have larger diameters are usually thin and long, so the average distance measure is quite likely to be misleading. Therefore, neither of these metric can be used to derive a density estimate for trap coverage.

In summary, there does not exist any work that can be used to derive estimates of density (or even critical conditions) needed in a random deployment to achieve trap coverage of a given diameter, a mathematically challenging problem that we address comprehensively in this paper. Similarly, although there have been numerous work on deterministic deployment patterns for full coverage and connectivity [2], none of them are applicable directly to Trap Coverage since this is a new model of coverage that allows holes of bounded diameter. We postpone discussing existing work related to algorithmic determination of the status of trap coverage to Section VI-A. 


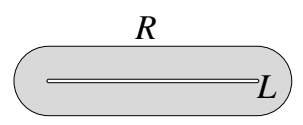

Fig. 2. Region $R$ and line $L$ in proof of lower bound on $\mathbb{P}\left(h_{m} \geq d\right)$. $L$ is uncovered and so forms a long thin hole provided $R$ is void of any sensors.

\section{Estimating THE DEnSity FOR RANDOM DEPLOYMENTS}

In this section, we derive a reliable estimate for density that will ensure trap coverage of a given diameter. We take a progressive approach in deriving our estimate for simplicity of exposition. We first consider a disk model of sensing. For this model, we first derive a crude but rigorous bound that may appeal to intuition. We then show that large holes occur with a Poisson distribution. In Section IV-A, we estimate the intensity of this Poisson distribution. Once we have an accurate estimate of the intensity with which large holes occur, we can accurately determine the density needed to achieve trap coverage of a given diameter $d$ with any given probability (such as with probability 0.9999). We show in Section VI-F that our density estimate is accurate even for small deployment regions, a significant improvement over asymptotic critical densities that work only for large deployments. Finally, we show in Section IV-B, how our derivations can be adapted to non-disk sensing models. We provide the derivation for randomly oriented ellipses as an example.

We consider a Poisson deployment with intensity $\lambda$ in a deployment region $\mathcal{A}^{\prime}$ that includes a large target region $\mathcal{A}$ of area $|\mathcal{A}|$. Write $n=\lambda|\mathcal{A}|$ for the expected number of sensors within the target region, and $h_{m}$ for the maximum hole diameter.

Before we obtain a bound on the probability that $h_{m} \geq d$, we make some remarks on the effect of the boundary. Generally speaking, if the deployment region $\mathcal{A}^{\prime}$ is the same as the target region $\mathcal{A}$, then coverage is more likely to fail at the boundary than in the interior (see [3]). Thus a similar result would be expected to occur for trap coverage, at least when $d / r$ is small. One simple way of avoiding problems at the boundary is to enlarge $\mathcal{A}^{\prime}$ so that it includes all points within distance $r$ of $\mathcal{A}$. (We shall assume in the following that the boundary of $\mathcal{A}$ is small, i.e., $|\partial \mathcal{A}|(r+d) \ll|\mathcal{A}|$. Thus enlarging the deployment region as above will not increase its area much, i.e., $\left|\mathcal{A}^{\prime}\right| /|\mathcal{A}| \approx 1$.) This makes coverage of points on the boundary of $\mathcal{A}$ as likely as points in the interior, and large holes are no more likely to appear at the boundary than in the interior (in fact less likely since there is less area near the boundary than the interior, and holes are confined to lie inside $\mathcal{A}$ ). In the following analysis we shall assume that the deployment region has been enlarged in this manner.

We first derive a lower bound on $\mathbb{P}\left(h_{m} \geq d\right)$. Let $L$ be a straight line of length $d$ inside $\mathcal{A}$. If there is no sensor within distance $r$ of $L$ then $L$ lies in the interior of a hole, which then must have diameter at least $d$. Let $R$ be the set of points within distance $r$ of $L$. Then $R$ consists of a $2 r \times d$ rectangle with two semicircular caps of radius $r$ attached to each end (see Figure 2). The probability that $R$ contains no sensor is
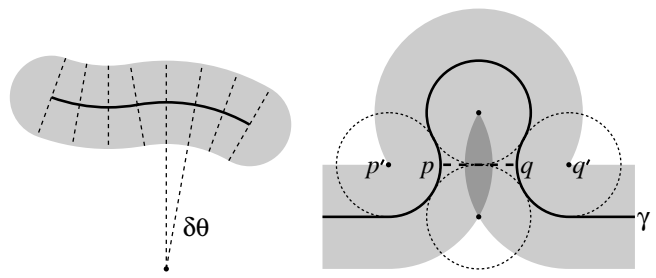

Fig. 3. Left: calculation of the area of $R_{\gamma}(s)$. Right: Example of self-overlapping $R_{\gamma}(s)$ with $s=r$. $R_{1}$ is lightly shaded region, $R_{2}$ is heavily shaded region. If $\gamma$ approaches within $2(\sqrt{3}-1) r>r$ of itself, then one can shorten $\gamma$ by cutting across along dashed line $p q$.

$e^{-\lambda|R|}$ where $|R|=2 r d+\pi r^{2}$. We can place $R$ inside a $2 r \times(d+2 r)$ rectangle which has area less than $2|R|$. Thus if $\mathcal{A}$ is large enough and of a reasonable shape (in particular, if it has small boundary as mentioned above), we can pack at least $|\mathcal{A}| /(2|R|)=n /(2 \lambda|R|)$ disjoint copies of $R$ into $\mathcal{A}$. The event that one copy of $R$ is devoid of sensors is independent of any of the other copies, so the probability that the maximum hole diameter is at least $d$ is bounded below by the probability that at least one of the copies of $R$ is empty. Thus

$$
\begin{aligned}
& \mathbb{P}\left(h_{m} \geq d\right) \geq 1-\left(1-e^{-\lambda|R|}\right)^{n /(2 \lambda|R|)} \geq 1-e^{-I|\mathcal{A}|}, \\
& \quad \text { where } \quad I=\left(2\left(2 r d+\pi r^{2}\right)\right)^{-1} e^{-\lambda\left(2 r d+\pi r^{2}\right)} .
\end{aligned}
$$

(Here we have used the fact that $1-x \leq e^{-x}$. The quantity $I$ is essentially a bound on the average number of holes of diameter $\geq d$ per unit area.) If we write

$$
\lambda\left(2 r d+\pi r^{2}\right)=\lambda|R|=\log n-\log \log n-t,
$$

then for $t=t(n)=o(\log n), I|\mathcal{A}|=\frac{e^{t} \log n}{2(\log n-\log \log n-t)}=$ $(.5+o(1)) e^{t}$. If $t \rightarrow \infty$ as $n \rightarrow \infty$ we have $I|\mathcal{A}| \rightarrow \infty$ and thus $\mathbb{P}\left(h_{m} \geq d\right) \rightarrow 1$.

Now, we give an upper bound on $\mathbb{P}\left(h_{m} \geq d\right)$, which is more involved. Suppose a hole $H$ of diameter $h_{m} \geq d$ exists. Suppose $x, y \in H$ are points with $\|x-y\|=d$ and let $\gamma$ be the shortest path from $x$ to $y$ inside the hole $H$. We may assume that $x$ lies at a crossing point of the boundaries of the sensing regions of two sensors (see Lemma 5.1 below). Note that $\gamma$ consists of straight line segments possibly joined together with arcs of circles of radius $r$. In particular, the radius of curvature of $\gamma$ at any point is never less than $r$.

Lemma 4.1: Suppose $0<s \leq r$. Then the set $R_{\gamma}(s)$ of points that lie within distance $s$ of $\gamma$ has area at least $s(|\gamma|+$ $d)+\pi s^{2}$, where $|\gamma| \geq d$ is the arc length of the curve $\gamma$.

Proof: Suppose first that $R_{\gamma}(s)$ does not wrap around on itself, i.e., no point on $\partial R_{\gamma}(s)$ is distance $s$ from more than one point of $\gamma$ (see Figure 3). Then the area of $R_{\gamma}(s)$ is exactly $2 s|\gamma|+\pi s^{2}$. To see this, cut $\gamma$ into small segments each of (approximately) constant radius of curvature, and make corresponding cuts in $R_{\gamma}(s)$ orthogonally to $\gamma$ at the places where $\gamma$ is cut. Suppose one segment of $\gamma$ has radius of curvature $R$ and subtends an angle $\delta \theta$. The length of this segment is $R \delta \theta$, while the area of the corresponding slice of $R_{\gamma}(s)$ is $\frac{1}{2}(R+s)^{2} \delta \theta-\frac{1}{2}(R-s)^{2} \delta \theta=2 s R \delta \theta$ (the difference between sectors of two disks). Adding up these areas for each segment of $\gamma$ gives an area of $2 s|\gamma|$, and adding the two halfdisks centered at the endpoints of $\gamma$ gives the result. 
Now assume $R_{\gamma}(s)$ self-intersects. Then the above argument will overestimate the area. However, distant parts of $\gamma$ cannot approach too closely. Indeed, suppose there are two points $p$ and $q$ on $\gamma$ such that $p \neq q$ and the distance between $p$ and $q$ is a local minimum for points on $\gamma$. Then there are sensors at $p^{\prime}, q^{\prime}$ with $p, q$ lying on the segment $p^{\prime} q^{\prime}$ and $\gamma$ following the boundaries of the sensor regions of $p^{\prime}$ and $q^{\prime}$ (see Figure 3). No sensor on the opposite side of $\gamma$ to $p^{\prime}$ and $q^{\prime}$ can have a sensor region intersecting the sensor regions of $p^{\prime}$ or $q^{\prime}$, but if $\left\|p^{\prime}-q^{\prime}\right\|<2 \sqrt{3} r$ this implies no sensor region intersects the line segment $p q$. Thus if $\|p-q\|<2(\sqrt{3}-1) r$ the line segment from $p$ to $q$ is uncovered by any sensor and $\gamma$ can be shortened by joining across from $p$ to $q$, contradicting the assumption that $\gamma$ was the shortest path from $x$ to $y$. A similar argument shows that no point can lie in a triple selfintersection of $R_{\gamma}(s)$. Indeed, if $w$ is such a point and $p_{1}$, $p_{2}, p_{3}$ are distinct locally closest points on $\gamma$, then there are sensors at $p_{i}^{\prime}$, where $p_{i}$ lies on the segment $w p_{i}^{\prime}$ and $\gamma$ follows the boundary of the sensor region of $p_{i}^{\prime}$ near $p_{i}$. If any of the distances $\left\|p_{i}^{\prime}-p_{j}^{\prime}\right\|, i \neq j$, are less than $2 \sqrt{3} r$, then $\gamma$ may be shortened. But if all $\left\|p_{i}^{\prime}-p_{j}^{\prime}\right\| \geq 2 \sqrt{3} r$ then their sensor regions do not intersect, and so $w$ does not exist.

Thus of the area $\left|R_{\gamma}(s)\right|$, no part can be more than double counted by the estimate $2 s|\gamma|+\pi s^{2}$ above. In other words, we can write $R_{\gamma}(s)$ as the union of two regions $R_{1}$ and $R_{2}$, with $\left|R_{1}\right|+2\left|R_{2}\right|=2 s|\gamma|+\pi s^{2}$. Now any line $L$ perpendicular to $x y$ between $x$ and $y$ must intersect $R_{1}$ in line segments of total length at least $2 s$ since no point on $L$ before the first point of $\gamma$ or after the last point of $\gamma$ can be in a selfintersection of $R_{\gamma}(s)$. Also $R_{1}$ contains two half-disks at $x$ and $y$. Thus $\left|R_{1}\right| \geq 2 s d+\pi s^{2}$ and $\left|R_{\gamma}(s)\right|=\left|R_{1}\right|+\left|R_{2}\right|=$ $\left|R_{1}\right| / 2+\left(\left|R_{1}\right|+2\left|R_{2}\right|\right) / 2 \geq s(|\gamma|+d)+\pi s^{2}$ as required.

Now approximate $\gamma$ with a path $\gamma^{\prime}$ that is made up from a sequence of arcs of circles, each of radius $r / 2$ and length $r \varepsilon$ (so they curve by an angle of $2 \varepsilon$ ). Each arc curves either to the left or the right. One can show that $\gamma^{\prime}$ can be chosen so that it starts at $x$, the angle that $\gamma^{\prime}$ makes with the horizontal at $x$ is a multiple of $\varepsilon$, and all points of $\gamma^{\prime}$ are within distance $C r \varepsilon^{2}$ of $\gamma$, where $C$ is some absolute constant. Hence there is no sensor within distance $r\left(1-C \varepsilon^{2}\right)$ of $\gamma^{\prime}$.

Given $x$, there are $(2 \pi / \varepsilon) 2^{k}$ choices for $\gamma^{\prime}$ when $\gamma^{\prime}$ consists of $k$ segments. Given $\gamma^{\prime}$, one knows $\gamma$ to within distance $C r \varepsilon^{2}$, so picking any $\gamma$ consistent with $\gamma^{\prime}$, we know $R_{\gamma}\left(r\left(1-2 C \varepsilon^{2}\right)\right)$ contains no sensors. Since the length of $\gamma$ and $\gamma^{\prime}$ agree to within a factor of $1+O\left(\varepsilon^{2}\right)$, any $\gamma^{\prime}$ gives us a region of area $\left(r^{2} k \varepsilon+r d+\pi r^{2}\right)\left(1-C^{\prime} \varepsilon^{2}\right)$ devoid of sensors, so the probability of some such $\gamma^{\prime}$ existing starting from $x$ is at most

$$
\begin{aligned}
& \sum_{k \geq d / r \varepsilon}(2 \pi / \varepsilon) 2^{k} e^{-\lambda\left(r^{2} k \varepsilon+r d+\pi r^{2}\right)\left(1-C^{\prime} \varepsilon^{2}\right)} \\
& \quad \leq \frac{2 \pi}{\varepsilon\left(1-2 e^{-\lambda r^{2} \varepsilon / 2}\right)} e^{-\lambda\left(2 r d+\pi r^{2}\right)\left(1-C^{\prime} \varepsilon^{2}\right)+(d / r \varepsilon) \log 2}
\end{aligned}
$$

Setting $\varepsilon=\left(\lambda r^{2}\right)^{-2 / 3}$ and assuming $\lambda r^{2} \gg 1$, this is at most

$$
C^{\prime \prime}\left(\lambda r^{2}\right)^{2 / 3} e^{-\lambda\left(2 r d+\pi r^{2}\right)\left(1-O\left(\left(\lambda r^{2}\right)^{-2 / 3}\right)\right.} .
$$

The expected number of intersection points in $\mathcal{A}$ we can choose for $x$ is $4 \lambda \pi r^{2} n$, so we obtain

$$
\mathbb{P}\left(h_{m} \geq d\right) \leq C^{\prime \prime \prime}\left(\lambda r^{2}\right)^{5 / 3} n e^{-\lambda\left(2 r d+\pi r^{2}\right)\left(1-O\left(\left(\lambda r^{2}\right)^{-2 / 3}\right)\right.}
$$

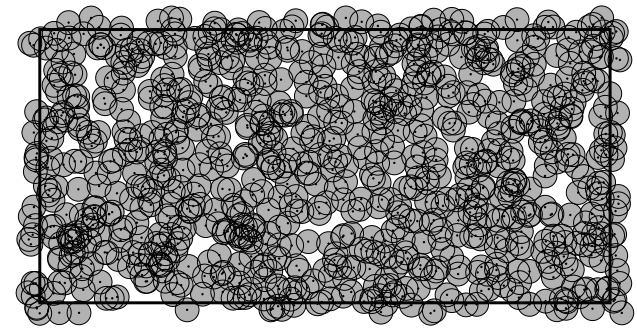

Fig. 4. Example of Poisson deployment. Rectangle denotes target region. Notice that holes of larger diameters are typically long and thin, although this need not be true for smaller diameter holes.

for some constant $C^{\prime \prime \prime}$. For $\lambda r^{2}=O(\log n)$, this tends to 0 when

$$
\lambda\left(2 r d+\pi r^{2}\right)\left(1-O\left(\left(\lambda r^{2}\right)^{-2 / 3}\right)\right) \geq \log n+O(\log \log n) .
$$

Combining this with the lower bound (3) above, we see that the maximum hole size $h_{m}=d$ typically occurs when

$$
\lambda\left(2 r d+\pi r^{2}\right)\left(1-O\left(\left(\lambda r^{2}\right)^{-2 / 3}\right)\right)=\log n,
$$

(the $O\left(\left(\lambda r^{2}\right)^{-2 / 3}\right)$ error term swallowing the $\log \log n$ terms in both cases). We observe that (from both the lower and upper bounds above) the holes with the largest diameter are long and thin, basically being obtained by insisting that an almost straight path $\gamma$ of length $d$ is not covered by any sensing region. We show in Figure 4, a representative Poisson deployment for which some holes exist. Note that although the holes are of various shapes, the holes with the largest diameters are usually "long and thin", confirming our analytical conclusion.

Comparison with an obvious extension of the full coverage model. Note that our estimate is significantly better than the naïve bound obtained by increasing $r$ by $d$ and then demanding that this provides full coverage. Indeed, our bound (assuming $\lambda r^{2} \gg 1$ ) is of the form

$$
\lambda\left(2 r d+\pi r^{2}\right) \approx \log n
$$

while if we required full coverage with sensing range $r+d$ we would need (replacing $d$ by 0 and $r$ by $r+d$ in (7))

$$
\lambda \pi(r+d)^{2}=\lambda\left(\pi d^{2}+2 \pi r d+\pi r^{2}\right) \approx \log n .
$$

Even for small $d$ we would underestimate $d$ by a factor of $\pi$

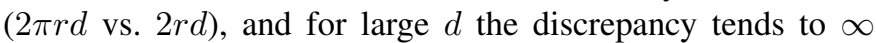
$\left(d \sim c \sqrt{\log n}\right.$ vs. $d \sim c r^{-1} \log n$ for fixed $\lambda$ ). Note that enlarging the sensor range by $d / 2$ is not sufficient in general to eliminate all holes of diameter $d$, but even if it were, the (incorrect) bound obtained on $d$ would still always be worse than our result. The reason for the discrepancy between our estimate and the naïve bound however becomes clear when we observe that a long thin hole can be covered with just a small increase in $r$, rather than increasing it by $d$.

Estimating the Probability Distribution of Large Holes. Large holes, when they exist, should be well separated, so one would expect the distribution of the number of holes with diameter $\geq d$ to follow an approximately Poisson distribution. This is indeed true for large $\lambda r^{2}$. To show this, suppose $H$ is a 
coverage hole. Then $H$ depends on the Poisson process within a region $H^{\prime}$ consisting of all points at distance $\leq r$ from $H$. To show the number of holes is approximately Poisson, one can use the Stein-Chen method (see [1]). In our case, it reduces to showing (a) that the expected number of pairs of holes $H_{1}$ and $H_{2}$ for which $H_{1}^{\prime}$ and $H_{2}^{\prime}$ intersect is $o(1)$, and (b) that this would also be true if the $H_{i}^{\prime}$ were truly independent. Condition (b) is easy to show since the $H_{i}^{\prime}$ are much smaller than $\mathcal{A}$. Condition (a) holds since conditioned of the state of the Poisson process in $H_{1}^{\prime}$, it is unlikely there is a hole close by. (Effectively this reduces to showing holes are rarely near the boundary of a deployment region $\mathbb{R}^{2} \backslash H_{1}^{\prime}$, which holds since the boundary of $H_{1}^{\prime}$ is typically not large.) We refer the reader to [4] for more details of these calculations. As a result, for sufficiently large $\lambda \pi r^{2}$

$$
\begin{gathered}
\mathbb{P}\left(h_{m} \geq d\right) \approx 1-e^{-I|\mathcal{A}|}, \text { where } \\
I=\lambda e^{-\lambda\left(2 r d+\pi r^{2}\right)\left(1-O\left(\left(\lambda r^{2}\right)^{-2 / 3}\right)\right.},
\end{gathered}
$$

$I$ being the expected number of holes of diameter at least $d$ per unit area (i.e., the intensity of the Poisson process for the occurrence of holes of diameter $\geq d$ ). Once again the $O()$ error term in $I$ swallows the polynomial factors in front of the exponentials in the upper and lower bounds given above. We shall refine this estimate in the next section.

\section{A. Refining the Estimate}

In this section we shall give a much more accurate estimate for the probability of occurrence of holes of diameter $\geq d$. We only provide an outline of our derivation here and defer the detailed proofs to [4]. To obtain an improved estimate, we compare the trap coverage model with that of barrier coverage, where sensors are deployed in a long (but 2 dimensional) horizontal rectangular strip $S_{h}$ of height $h$, and one asks whether there are coverage holes crossing the strip (see [3] for details). We shall count the number of holes that cut across this strip in two different ways, leading to a comparison between barrier coverage and trap coverage. First let $I_{d}^{\text {trap }}$ be the number of holes of diameter at least $d$ per unit area and assume $u, v$ are endpoints of such a hole with $u$ lying below $v$. Then since the holes are typically long and thin, this hole will cut across $S_{h}$ provided $u$ and $v$ lie on opposite sides of $S_{h}$. Let $\theta$ be the angle $u v$ makes with the vertical, and $x$ the distance of $u$ below the bottom of $S_{h}$ (see Figure 5). Then we need $\|u-v\| \geq(x+h) / \cos \theta$. The intensity $I$ of such holes per unit length along $S_{h}$ is therefore given approximately by

$$
I \approx \frac{1}{\pi} \int_{-\pi / 2}^{\pi / 2} \int_{0}^{\infty} I_{(x+h) / \cos \theta}^{\mathrm{trap}} d x d \theta .
$$

To relate this to $I_{d}^{\text {trap }}$ at a particular value of $d$, we note that by our simple estimates in the previous section that $I_{d}^{\text {trap }}$ decays exponentially with $d$,

$$
I_{d+\varepsilon}^{\text {trap }} \approx I_{d}^{\text {trap }} e^{-2 \lambda r \varepsilon}
$$

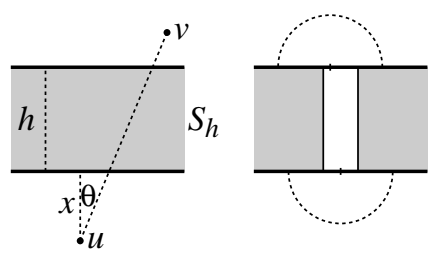

Fig. 5. Left: hole with diameter $u v$ crossing strip $S_{h}$. Right: additional vacant semicircular areas allow break to form hole.

Using this approximation (and evaluating the $x$-integral) gives

$$
\begin{aligned}
I & \approx I_{h}^{\text {trap }} \frac{1}{2 \pi \lambda r} \int_{-\pi / 2}^{\pi / 2} e^{-2 \lambda r h(1 / \cos \theta-1)} \cos \theta d \theta \\
& \approx I_{h}^{\text {trap }}\left(4 \pi \lambda^{2} r^{2}\left(\lambda r h+\frac{9}{8}\right)\right)^{-1 / 2}
\end{aligned}
$$

where the last approximation is valid for large $\lambda r h$.

Now we evaluate $I$ by comparison with barrier coverage. A hole across $S_{h}$ results in a break as defined in [3], however when defining barrier coverage one assumes deployment only inside the strip $S_{h}$. Thus for a break to define a hole crossing $S_{h}$, we also need that sensors outside of $S_{h}$ do not destroy the break. From the results in [3] we know that most breaks are approximately rectangular and thin cutting perpendicularly across $S_{h}$. Using this it follows that for this break to make a hole, one needs at least one point on the top boundary of $S_{h}$ inside the break to be uncovered by sensors outside of $S_{h}$, and similarly at least one point on the bottom boundary of $S_{h}$ to be uncovered (see Figure 5). One can show that the probability of some point on the top boundary of $S_{h}$ in a fixed interval of length $W$ to be uncovered by sensors above $S_{h}$ is approximately $(1+\lambda r W) e^{-\pi \lambda r^{2} / 2}$. One may assume the top and bottom boundaries are independent for large $h$ (in fact $\lambda h^{3} \gg r$ is enough), so this gives

$$
I \approx I_{h}^{\text {barrier }}(1+\lambda r \mathbb{E}(W))^{2} e^{-\pi \lambda r^{2}},
$$

where $\mathbb{E}(W)$ is the expected width of the uncovered interval on the boundary of $S_{h}$ that occurs at a break, and $I_{h}^{\text {barrier }}$ is the average number of breaks per unit distance along $S_{h}$. One can show using the techniques of [3] that $\mathbb{E}(W) \sim c \lambda^{-2 / 3} r^{-1 / 3}$ with $c \approx 0.72$. Also [3] gives the following estimate for $I_{h}^{\text {barrier }}$.

$$
I_{h}^{\text {barrier }} \approx \lambda^{2 / 3}(2 r)^{1 / 3} e^{-2 \lambda r d\left(1-\alpha\left(4 \lambda r^{2}\right)^{-2 / 3}\right)+\beta} .
$$

where $\alpha \approx 1.12794$ and $\beta \approx-1.05116$. (Note that the value of $r$ in [3] is twice the sensor radius.) Putting these together gives the following approximation for $I_{d}^{\text {trap }}$.

$$
\begin{aligned}
I_{d}^{\text {trap } \approx} & C_{0} \lambda\left(\lambda r^{2}\right)^{2 / 3}\left(1+c\left(\lambda r^{2}\right)^{1 / 3}\right)^{2}\left(\lambda r d+\frac{9}{8}\right)^{1 / 2} \\
& \times e^{-2 \lambda r d\left(1-\alpha\left(4 \lambda r^{2}\right)^{-2 / 3}\right)-\pi \lambda r^{2}}
\end{aligned}
$$

where $C_{0}=\pi^{1 / 2} 2^{4 / 3} e^{\beta} \approx 1.5611, \alpha \approx 1.12794, c \approx 0.72$. As in [3], this estimate should be valid for $\lambda d^{3} \gg r$, and $\lambda r^{2} \gg 1$, which in our context means not too close to either full coverage $\pi \lambda r^{2} \sim \log n$ or the percolation threshold $\lambda r^{2} \sim$ constant.

Since coverage holes of diameter $\geq d$ follow Poisson distribution (using the same Stein-Chen argument as in the previous section), we have

$$
\mathbb{P}\left(h_{m} \geq d\right) \approx 1-e^{-|\mathcal{A}| I_{d}^{\text {trap }}}
$$


when $I_{d}^{\text {trap }}$ is small.

\section{B. Extending to Non-disk Sensing Regions}

The above analysis assumes that the sensing regions are disks. However, it is clear from the lower bound argument for $\mathbb{P}\left(h_{m} \geq d\right)$ that we can generalize this to other shapes of sensing region. To recall, for the lower bound we require that no sensing region intersects a line $L$. The probability that this occurs can be calculated for any required (even probabilistic) model of the sensing region. The fact that the upper bound for disks is close to the lower bound suggests that this will also hold for most reasonably "disk-like" sensing regions. As an example, we consider the case of randomly oriented ellipses (to model biased gain along a randomly oriented axis).

Lemma 4.2: Suppose the sensing regions are ellipses, each with maximum and minimum radii $r$ and $\alpha r$ respectively, and with orientation that is random and uniform. Then the expected number of sensor regions meeting a fixed line $L$ of length $d$ is given exactly by

$$
\lambda\left(\pi r^{2} \alpha+2 r d \frac{2}{\pi} E\left(1-\alpha^{2}\right)\right),
$$

where $E(m)=\int_{0}^{\pi / 2}\left(1-m \sin ^{2} \theta\right)^{1 / 2}$ is an elliptic integral.

Proof: Consider the sensors whose smaller radius lies in some small angle $[\theta, \theta+d \theta]$ from the direction of the line $L$. These sensors occur as a Poisson process of intensity $\frac{\lambda}{2 \pi} d \theta$. If we scale the plane by stretching by a factor $1 / \alpha$ in the direction of the smaller radius, the sensor regions become circular with radius $r$, while the density of sensors is now $\alpha \frac{\lambda}{2 \pi} d \theta$. The line $L$ is now also stretched, and has a new length $d^{\prime}=d\left(\alpha^{-2} \cos ^{2} \theta+\sin ^{2} \theta\right)^{1 / 2}$. The expected number of these sensors meeting $L$ is therefore equal to

$$
\begin{gathered}
\left(\pi r^{2}+2 r d^{\prime}\right) \alpha \frac{\lambda}{2 \pi} d \theta \\
=\frac{\lambda}{2 \pi}\left(\pi r^{2} \alpha+2 r d\left(1-\left(1-\alpha^{2}\right) \sin ^{2} \theta\right)^{1 / 2}\right) d \theta .
\end{gathered}
$$

The result follows by integrating this from $\theta=0$ to $2 \pi$.

Note that since we are assuming Poisson deployment, the physical location of the sensor within the ellipse is irrelevant (as long as it is independent of the location and orientation of the ellipse), so we may for example assume the sensor is at the center, or at a focal point, or at one end of the ellipse. The results will be identical in all cases. The lower bound argument for $\mathbb{P}\left(h_{m} \geq d\right)$ follows exactly as before, using (11) in place of the expression $\lambda|R|=\lambda\left(\pi r^{2}+2 r d\right)$. Similarly, the upper bound argument also follows, except that the radii of curvature of the path $\gamma$ may need to be reduced, leading to worse constants in the $O()$ term in (5) when $\alpha$ is small.

Similar results can be shown for probabilistic sensing regions. For example, if the radii $r$ varied randomly then one obtains the same results with $\lambda|R|$ replaced with $\mathbb{E} \lambda|R|=$ $\lambda\left(\pi \mathbb{E}\left(r^{2}\right)+2 \mathbb{E}(r) d\right)$ (for the disk model), provided the random radii $r$ is is bounded, $r_{1}<r<r_{2}$, and with the error terms depending on $r_{1}$ and $r_{2}$.

\section{Optimal Patterns for Deterministic DEPLOYMENTS}

In this section, we address the problem of finding the optimal patterns for distributing sensor nodes deterministically
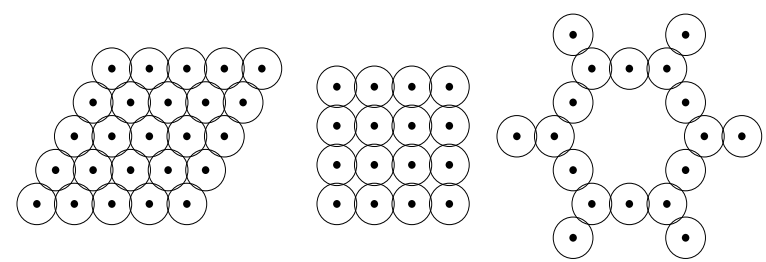

Fig. 6. Conjectural optimal deployments - triangular grid $(d \lesssim$ $1.1292 r$ ), square grid $(1.1292 r \lesssim d \lesssim 2.0798 r$ ), and (possibly subdivided) hexagonal grid $(d \gtrsim 2.0798 r)$. The triangular and square grids for $r \leq d \lesssim 1.1292 r$, respectively $2 r \leq d \lesssim 2.0798 r$, are such that the sensing regions just touch, and in fact have trap coverage diameter $r$ and $2 r$.

to achieve $d$-Trap Coverage for a given diameter $d$. Throughout we shall assume all sensors have disk sensing regions of radius $r$.

Hunting for optimal deployment patterns has intrigued mathematician and computer scientists for decades, and many problems remain unsolved in general. While for the full coverage model, it is know that triangular lattice is optimal, the same problem is much harder for the Trap Coverage model, which reduces to the full coverage model only when $d=0$.

Our aim is to determine a deployment pattern which gives a fixed bound $d$ on the trap coverage diameter, while minimizing the number of sensors required. We consider the dimensions of the deployment region to be large, tending to infinity. We show that for $d \leq 0.5552 r$, the optimal deployment pattern (in this limit) is a triangular grid. We conjecture that this is in fact optimal for all $d \lesssim 1.1292 r$, a square grid is optimal for $1.1292 r \lesssim d \lesssim 2.0798 r$, and a hexagonal grid is optimal for larger $d$, where we may subdivide the edges of the hexagonal grid a number of times if $d$ is large (see Figure 6). For all $d$ we give good bounds on the optimal deployment density, showing in particular that the subdivided hexagonal grid is within $10 \%$ of optimal for large $d / r$. However, proving the exact optimal pattern appears to be an extremely difficult problem, related to several open problems in optimal plane packing. Note that for any practical surveillance or monitoring applications a large $d / r$ ratio is a realistic assumption.

\section{A. Assumptions and Definitions}

Let $\mathcal{A}$ denote the target region (as in Section IV) and $S$ the set of sensors deployed. For simplicity and to avoid boundary effects we shall assume $\mathcal{A}$ is a large (2-dimensional) torus. All the results in this report can be extended to the case when $\mathcal{A}$ is a finite regions in the plane, with error bounds depending on the ratio of the boundary length to the area. As a consequence, our results also apply in the limit of large, reasonably shaped, planar deployment regions as the area of the deployment region tends to infinity. A deployment on a torus is also equivalent to an infinite deployment in the plane which is periodic in two directions.

We shall assume each sensor $x \in S$ has a sensing region $S_{x}$ which is an open disk of radius $r$ about $s \in \mathcal{A}$. We shall assume that the dimensions of the torus are such that the shortest non-contractible curve in $\mathcal{A}$ has length large 
compared with $r+d$ so as to avoid sensor regions overlapping themselves, or sensor coverage holes wrapping round the torus.

Let $C_{S}=\bigcup_{x \in S} S_{x}$ denote the region covered by $S$, that is, the union of the sensing regions of the nodes in $S$, and $U_{S}$ denote the uncovered region in $\mathcal{A}$, that is, $U_{S}=\mathcal{A} \backslash C_{S}$. A coverage hole is a connected component of $U_{S}$. The sensor network is said to provide trap coverage with diameter $d$ if the diameter of any coverage hole in $\mathcal{A}$ is at most $d$.

A sensing neighbor of a sensor $x \in S$ is a sensor $y \in S$ whose sensing region overlaps with the sensing region of $s$, i.e., $S_{y} \cap S_{x} \neq \emptyset$. Consider the perimeter of the sensing region of $x$, which is divided into one or more segments by the sensing perimeters of its sensing neighbors. Every such segment is called a sensing segment of $x$. Note that the boundary of a coverage hole is made up of a number of sensing segments.

\section{B. The Delauney graph $H_{S}$}

Suppose we have a deployment of a finite number of sensors in the torus $\mathcal{A}$. We construct the coverage graph $G_{S}=(S, E)$ by taking the sensors as the vertices and joining sensor neighbors, i.e., we join vertices with an edge if and only if the sensors are within distance $2 r$ of each other so that their sensing regions intersect. We represent the edges as straight line segments joining the vertices, so that the edges lie entirely within the sensing region. By assumption on the dimensions of $\mathcal{A}$, this line segment is unique. Note that edges of $G_{S}$ may cross each other. For the analysis of the deployment we shall consider a certain spanning subgraph $H_{S}$ of $G_{S}$ that has no crossing edges, so is 'planar', and for which each coverage hole corresponds naturally to certain faces of $H_{S}$.

The graph $H_{S}$ will be a slightly modified version of the Delauney graph of the set of sensors. For each sensor $x$, let $V_{x}$ be the set of points covered by the sensing region that are no further from $x$ than from any other sensor:

$V_{x}=\left\{z \in \mathbb{R}^{2}:\|z-x\| \leq r\right.$ and $\left.\forall y \in V:\|z-x\| \leq\|z-y\|\right\}$.

Here $\|z-x\|$ denotes the shortest distance between $z$ and $x$ on the torus. Thus $V_{x}$ is the Voronoi cell of $x$, except that we do not include points at distance more than $r$ from $x$. Equivalently, it is the intersection of the Voronoi cell of $x$ with the coverage region. (If $z$ is in the coverage region then clearly the closed sensor to it is within distance $r$.) The edges of $H$ are given by pairs of sensors $x, y$ such that the Voronoi cells of $x$ and $y$ meet. We shall assume that no point is in more than three Voronoi cells. If this occurs we break ties arbitrarily, removing this point from all but three Voronoi cells. (For example, we can move each sensor in $S$ a small random distance, so that the existence of a point equidistance from four or more sensors occurs with probability zero.) Then $H_{S}$ will have no crossing edges. Indeed, it is a subgraph of the classical planar Delauney triangulation, where we have just removed edges (where the full Voronoi cells would have intersected, but did not because we truncated the Voronoi cells at distance $r$ ). Note that this is not quite the same as the Delauney triangulation with edges of length $>2 r$ deleted - edges of length $<2 r$ in the Delauney triangulation may correspond to Voronoi cells that intersect at distance $>r$ from their respective centers.

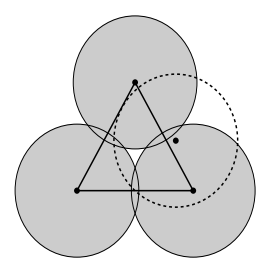

Fig. 7. Induced hole (dashed triangle) formed by triangle which is covered by a sensor disk of a sensor outside of the triangular face.

Suppose we are given any subgraph $H$ of $G_{S}$ in which edges do not cross. Then $H$ divides the torus $\mathcal{A}$ into faces. As above, a coverage hole is any connected component of the uncovered region in the torus. We define an induced hole of a face $f$ of $H$ to be a component of the subset of the face that is uncovered by the vertices on the boundary of that face. Note that a face may have several induced holes, or none at all, and an induced hole may be partially or entirely covered by sensors that lie outside the boundary of the face (see Figure 7). However, this will not occur for our graph $H_{S}$.

Lemma 5.1: Assume that the coverage region $C_{S}$ is connected and $S$ provides trap coverage with diameter $d$. Then $H_{S}$ is connected and every face $f$ of $H_{S}$ satisfies the following properties.

(a) there is at most one induced hole of $f$;

(b) if $f$ has no induced hole then $f$ is a triangle;

(c) if $f$ has an induced hole then it is a hole of $G$;

(d) if there is an induce hole of $f$ then each vertex on the boundary of $f$ is at distance $r$ from this induced hole (so the boundary of the induced hole includes a segment on the boundary of the sensing region of this vertex).

Proof: The coverage region is the union of the $V_{x}$, so this is connected iff $H_{S}$ is connected. Thus is only remains to prove that every face of $H_{S}$ satisfies (a)-(d).

Note first that each $V_{x}$ is convex, indeed, it it the intersection of the Voronoi cell of $x$ with the disk of radius of $r$ about $x$, both of which are convex. Suppose $f$ is a face of $H_{S}$, and $v_{1}, \ldots, v_{n}$ are the vertices (sensors) on the boundary of $f$ in that order, possibly with repeats. Then the Voronoi cell of $v_{i}$ intersects with that of $v_{i+1}$. Thus there is a (topological) closed curve $\gamma$ that goes through each $v_{i}$ in turn and stays within the Voronoi cells of $v_{i}$ and $v_{i+1}$ as it travels from $v_{i}$ to $v_{i+1}$. Now let $z$ be a covered point with in face $f$. Then $z$ lies in some $V_{x}$. If $z$ lies in the interior of $\gamma$ then $x$ must be one of the $v_{i}$ as $V_{x}$ cannot cross $\gamma$ and $x$ cannot lie inside the face $f$. If $z$ lies outside $\gamma$ but inside $f$, then it is trapped between an edge $v_{i} v_{i+1}$ of $f$ and the corresponding subpath $\gamma^{\prime}$ of $\gamma$ between $v_{i}$ and $v_{i+1}$. However every point of $\gamma^{\prime}$ is either within $r$ of $v_{i}$ or within $r$ of $v_{i+1}$, and moving closer to $v_{i} v_{i+1}$ perpendicular to $v_{i} v_{i+1}$ decreases these distances. Thus every point between $\gamma^{\prime}$ and the line segment $v_{i} v_{i+1}$ is within $r$ of either $v_{i}$ or $v_{i+1}$. Thus every covered point $z$ inside $f$ is covered by some $v_{i}$. Thus (c) holds.

Suppose $U$ is a coverage hole. Then due to trap coverage, connectivity of $C_{S}$, and the assumptions on $\mathcal{A}$, the boundary $\gamma$ of $U$ must be a simple closed curve. This boundary is covered by Voronoi cells $V_{v_{1}}, \ldots, V_{v_{n}}$ (in that order). But then $v_{i}$ is 
joined to $v_{i+1}$ in $H_{S}$, so we have a cycle $C=\left(v_{1}, \ldots, v_{n}\right)$ in $H_{S}$. As above, every point lying between $\gamma$ and the polygon $C$ must be covered by one of the $v_{i}$. Thus there are no other sensors inside $C$, and any other coverage hole must lie outside of $C$. Thus the face containing $U$ is $C$, and $C$ contains no other coverage hole. Hence (a) holds.

Now suppose $f$ is a face with boundary $C=\left(v_{1}, \ldots, v_{n}\right)$ and suppose $V_{v_{2}}$, say, does not meet any induced hole in $f$. As above, let $\gamma$ be a (topological) closed curve through the $v_{i}$, passing through each $V_{v_{i}}$ in turn. As one traverses the boundary of $V_{v_{2}}$ from the point where $\gamma$ meets $V_{v_{1}} \cap V_{v_{2}}$ to the point where $\gamma$ meets $V_{v_{3}} \cap V_{v_{2}}$ (staying in the interior of $\gamma$ ), one must come to a point $P$ that either lies in $V_{v_{2}} \cap V_{v_{3}}$, or lies in neither $V_{v_{2}}$ or $V_{v_{3}}$. In the first case $v_{2} v_{3}$ is an edge of $H_{S}$, so $C$ is a triangle. In the second case, either $P$ is $\mathrm{n}$ the boundary of an induced hole, or $P \in V_{v_{i}}$ for some $i \neq 1,2,3$. But in this last case $v_{i} v_{1}$ is an edge of $C$, a contradiction. Thus either $C$ is a triangle, or $C$ has an induced hole. Thus (b) holds. Finally, we know from the above argument that either $C$ is a triangle or $V_{v_{2}}$ meets the induced hole. But in this case $v_{2}$ must be at distance $r$ from the hole. As this applies to any vertex of $C$, either (d) holds or $C$ is a triangle. However any induced hole must have at least three sensor segments on its boundary, so (d) automatically holds for triangular faces.

Corollary 5.1: If $S$ provides trap coverage with diameter $d$ then the diameter of any face of $H_{S}$ is at most $d+2 r$.

Proof: The diameter of a face $f$ is the maximum distance between corners. If $f$ has an induced hole then every corner is within distance $r$ of this induced hole, and this induced hole is a coverage hole. Thus the distance between these two corners is at most $r+d+r=d+2 r$. If $f$ does not have an induced hole then it is a triangle with edge length at most $2 r$. Thus its diameter is at most $2 r \leq d+2 r$.

Lemma 5.2: Assume $S$ is an optimal deployment, i.e., a deployment of minimum size $|S|$ which achieves trap coverage with diameter $d$. Then $C_{S}$ is connected, and every face of $H_{S}$ is a proper cycle (without repeated vertices).

Proof: Suppose first that $C_{S}$ is disconnected. As each coverage hole has diameter at most $d$, which is much smaller than the dimensions of the torus $\mathcal{A}$, one cannot have two large components. Indeed, all but one component must lie inside a surrounding hole $h$. Now $h$ is bounded by an outer hole boundary consisting of sensing segments $s_{1}, \ldots, s_{n}$. Such a boundary is a simple closed curve and encloses the hole. Note that the diameter of the hole is the same as the maximum distance between any two points both of which are endpoints of the $s_{i}$. This follows from the fact that the convex hull of the hole boundary has these points as its corners. Removing any component inside the hole does not change the diameter of $h$, and so does not violate trap coverage. Since removing the component reduces $|S|$ and $S$ was assumed optimal, we conclude that $C_{S}$ is connected.

Now consider a hole $h$ with boundary $s_{1}, \ldots, s_{n}$ as above. The boundary gives rise to a cycle of sensors $C_{h}=$ $\left(x_{1}, \ldots, x_{n}\right)$, where $s_{i}$ is a sensing segment of sensor $x_{i}$. Note that $C_{h}$ is the boundary of the face of $H_{S}$ that contains $h$. Suppose that this cycle repeats a sensor, say $x_{i}=x_{j}=x$, $i<j$. Let $s_{i}$ be the sensing segment from point $p_{-}$to $p_{+}$

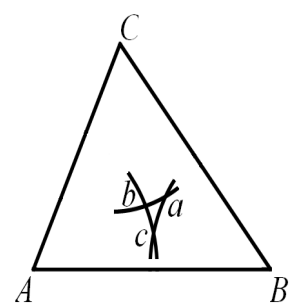

Fig. 8. Moving $C$ horizontally minimizes diameter of hole when $A C=B C$.

around the sensing disk of $x$, and let $s_{j}$ be the sensing segment from $q_{-}$to $q_{+}$, where we use a clockwise ordering of the points around the hole boundary (i.e., the hole boundary encounters the points in the order $p_{-}, p_{+}, q_{-}, q_{+}$when traversed in a clockwise orientation). the sensing segments $s_{i}$ and $s_{j}$ do not intersect as they are non-adjacent segments of the hole boundary. Thus the points occur in the order $p_{-}, p_{+}, q_{-}, q_{+}$ when traversing the boundary of the sensor region of $x$ counterclockwise. By removing the segments $s_{i}$ and $s_{j}$ from the sensor hole and adding the straight line segments $p_{-} q_{+}$ and $p_{+} q_{-}$one obtains two simple closed curves that do not intersect. Thus one curve encloses the other, wlog this one is $s_{1} \ldots s_{i-1} p_{-} q_{+} s_{j+1} \ldots s_{n}$. The diameter of the outermost curve is still the diameter of the hole, so removing sensors $x_{i+1}, \ldots, x_{j-1}$ does not increase the maximum hole diameter. Thus we may assume that all sensors $x_{1}, \ldots, x_{n}$ are distinct.

\section{Optimal Deployments}

Let $V=|S|$ be the number of sensors and denote the area of $\mathcal{A}$ by $A$.

Theorem 5.1: The average area per sensor $A / V$ in a deployment with maximum hole diameter $d$ is bounded by the maximum of $\frac{3 \sqrt{3}}{2} r^{2}+\frac{3}{2} d\left(r^{2}-\frac{d^{2}}{4}\right)^{1 / 2}-\frac{\sqrt{3}}{4} d^{2}$ and $\frac{1}{2}(d+2 r)^{2}$.

Proof: Define $A_{f}$ to be the area and $n_{f}$ to be the number of sides of the face $f$ of $H_{S}$. Write $V, E$, and $F$ for the number of vertices, edges, and faces of $H$. Then $V$ is the total number of sensors, $\sum_{f} n_{f}=2 E$, and by Euler's formula (on a torus), $V=E-F$. Thus $V=\frac{1}{2} \sum_{f}\left(n_{f}-2\right)$ while $A=\sum_{f} A_{f}$. The ratio $A / V$ is at most the maximum of the individual ratios $2 A_{f} /\left(n_{f}-2\right)$, so a bound on $A / V$ can be obtained by maximizing the quantity $2 A_{f} /\left(n_{f}-2\right)$ over all polygons with $n_{f}$ vertices and area $A_{f}$ with induced hole of diameter at most $d$.

Suppose $A B C$ is a triangle with an induced hole $a b c$ where $|a B|=|a C|=r$, etc. (see Figure 8). Fix one side $A B$ of the triangle, and assume $A B$ is horizontal. Then if we move the third vertex $C$ horizontally, the area of $A B C$ is unchanged. However, it is not hard to see that each of $|a b|$, $|b c|$, and $|c a|$ are convex functions of the horizontal position of $C$. Since the diameter of the hole is the maximum of these, it is also convex, and by symmetry is minimized when $|A C|=|B C|$. Applying this argument to $A C$ varying $B$ instead shows that the diameter of the hole is minimized for an equilateral triangle. By scaling the triangle, this is equivalent to maximizing the area for a given diameter of 
hole, so the optimum occurs for an equilateral triangle. The formula $\frac{3 \sqrt{3}}{2} r^{2}+\frac{3}{2} d\left(r^{2}-\frac{d^{2}}{4}\right)^{1 / 2}-\frac{\sqrt{3}}{4} d^{2}$ for $2 A_{f} /\left(n_{f}-2\right)$ is an elementary calculation.

For faces with $n_{f}>3$ we note that the diameter $d_{f}$ of the face $f$ is the maximum distance between any two of its vertices. However, each vertex is at distance $r$ from the hole, so $d_{f} \leq d+2 r$. The problem of finding the largest area of a polygon with a given diameter (the biggest little polygon problem) is unsolved in general, but $\mathrm{K}$. Reinhardt proved in 1922 that regular polygons are optimal for all odd $n_{f}$, and also for $n_{f}=4$. The area of a square of diameter $d_{f}$ is $\frac{1}{2} d_{f}^{2}$ and that of a regular pentagon is about $0.66 d_{f}^{2}$. For larger $n_{f}$ we use the fact (from the isodiametric inequality in two dimensions) that the shape with the largest area and given diameter is a circle (although not all shapes with a given diameter can fit into such a circle, which is addressed in the Lebesgue's minimal problem). This gives a bound of $A_{f} \leq \frac{\pi}{4} d_{f}^{2}$. Thus in general for $n_{f}>3,2 A_{f} /\left(n_{f}-2\right) \leq \frac{1}{2} d_{f}^{2} \leq \frac{1}{2}(d+2 r)^{2}$.

The above theorem is not good for large $d / r$. In this case we can use the following.

Theorem 5.2: The average area per sensor $A / V$ in a deployment with maximum hole diameter $d$ is bounded as $A / V \leq r(d+2 r)^{2} / d$.

Proof: We use the fact that the circle is the shape with the largest area for a given perimeter. Thus the perimeter $p_{f}$ of $f$ must be at least $2\left(\pi A_{f}\right)^{1 / 2}$. Since each edge of $H$ is of length at most $2 r, p_{f} \leq 2 r n_{f}$, and so $n_{f} \geq\left(\pi A_{f} / r^{2}\right)^{1 / 2}$, and hence $2 A_{f} / n_{f} \leq\left(4 r^{2} A_{f} / \pi\right)^{1 / 2}$. But we also know that $A_{f} \leq \frac{\pi}{4} d_{f}^{2}$ where $d_{f}$ is the diameter of $f$. Thus $2 A_{f} / n_{f} \leq r d_{f}$. Since $2 r n_{f} \geq p_{f} \geq 2 d_{f}, 2 A_{f} /\left(n_{f}-2\right) \leq r d_{f}\left(d_{f} /\left(d_{f}-2 r\right)\right)=$ $r d_{f}^{2} /\left(d_{f}-2 r\right)$. For $d_{f} \geq 4 r$ this is maximized by taking $d_{f}$ as large as possible, namely $d+2 r$, and the result follows. For $d_{f}<4 r$ we may assume $n_{f}>3$ (since the bound for triangles above is always lower than $\left.r(d+2 r)^{2} / d\right)$. But then $2 A_{f} /\left(n_{f}-2\right) \leq r d_{f} n_{f} /\left(n_{f}-2\right) \leq 8 r^{2}$ is always less than $r(d+2 r)^{2} / d$. The second bound thus follows.

Corollary 5.2: For $d / r \leq 0.5552$, the optimal deployment pattern in the plane is a triangular lattice.

Proof: For $d / r \leq 0.5552$, the value of $2 A_{f} /\left(n_{f}-2\right)$ for an equilateral triangle is larger than the bound for polygons given in Theorem 5.1 for $n_{f}>3$. Since triangles tile the plane, we can make every face of $H$ a triangle, so the maximum value $A / V=2 A_{f} /\left(n_{f}-2\right)$ can be achieved.

As $d / r$ grows, the optimal value of $2 A_{f} /\left(n_{f}-2\right)$ occurs for polygons with more sides, however these do not necessarily tile the plane, so the corresponding value of $A / V$ may not be attained. We conjecture that the optimal deployment patterns are all either triangular, square, or (possibly subdivided) hexagonal grids. Figure 9 shows the conjectured optimal patterns for various values of $d / r$.

Theorem 5.3: For large $d / r$, the subdivided hexagonal lattice is within $10 \%$ of the density of the optimal deployment. More precisely, $A / V \leq\left(\sqrt[4]{\pi^{2} / 12}+O(\sqrt{r / d})\right) r d$.

Proof: Take an optimal configuration, construct the graph $H_{S}$ as above. Then each face of $H_{S}$ has maximum diameter at most $d+2 r$. Note that an face with at most $2 k-1$ sides must have diameter at most $2 r(k-1)$ as the diameter must be the distance between two vertices and the maximum distance

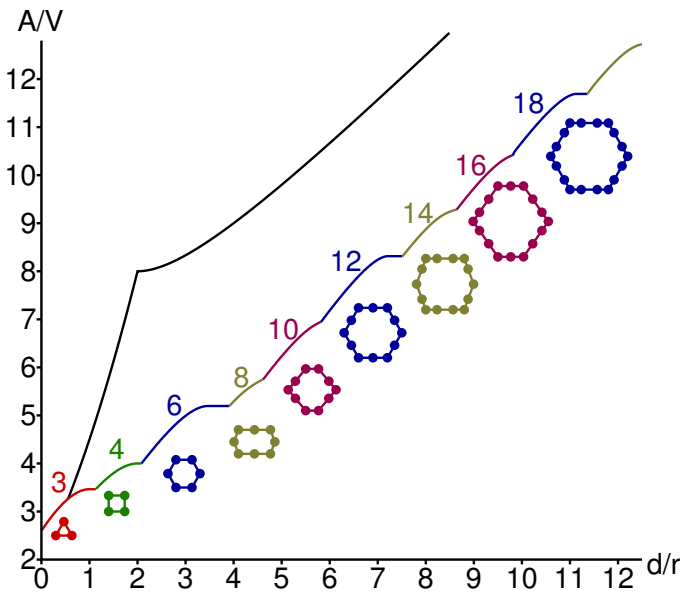

Fig. 9. Bound on ratio $A / V$ for optimal deployment with $r=1$ (upper curve), together will conjectured values (lower curve). The conjectured optimal deployment is either a triangular lattice, a square lattice, or a subdivided hexagonal lattice. Note that as the number of subdivisions of the hexagonal lattice increases, the exact shape of the hexagons changes, only being regular when the number of subdivisions on each side is equal. Also the subdivisions are not necessarily equally spaced along each edge.

between two vertices is at most $2 r(k-1)$. Remove edges between faces if both faces have $<2 k$ sides. Then if $4 r(k-$ $1)<d+2 r$, the diameter of any resulting face will still be at most $d+2 r$. Now amalgamate any remaining faces with $<2 k$ sides with some adjacent face (which must have $\geq 2 k$ sides). As any point is within distance $2 r(k-1)$ of a face with $\geq 2 k$ we see that the maximum diameter of any face is now at most $d+2 r+4 r(k-1)=d+2 r(2 k-1)$, and all faces have $\geq 2 k$ sides. the disk is the shape with the largest area for a given diameter, so the area of each face $f$ satisfies $A_{f} \leq A_{0}:=\frac{\pi}{4}(d+2 r(2 k-1))^{2}$. We now use the the result (Theorem 3 of [13]) that the sum $p$ of the edge lengths of the resulting graph is at least $\sqrt[4]{12} A / \sqrt{A_{0}}$, where $A$ is the sum of the area of the faces. (We need to scale all lengths by a factor $\sqrt{A_{0}}$, as this theorem assumes all areas are bounded by 1.) Thus

$$
\sum_{f} 2 r n_{f} \geq \sum p_{f} \geq 2 p \geq 2 \sqrt[4]{12} A / \sqrt{A_{0}},
$$

where $p_{f}$ is the perimeter of the face $f$. Note that each edge contributing to $p$ is counted in the perimeters of two faces. Now $n_{f} \geq 2 k$, so

$$
\sum 2 r\left(n_{f}-2\right) \geq \frac{k-1}{k} 2 \sqrt[4]{12} A / \sqrt{A_{0}} .
$$

Thus

$A / V=2 A / \sum\left(n_{f}-2\right) \leq \frac{k}{k-1} \frac{2 \sqrt{A_{0}}}{\sqrt[4]{12} r}=\sqrt[4]{\frac{\pi^{2}}{12}} \frac{k}{k-1}(d+2 r(2 k-1))$

whenever $4 r k<d$. Taking $k \approx \sqrt{d / r}$ gives

$$
A / V \leq \sqrt[4]{\frac{\pi^{2}}{12}}(1+O(\sqrt{r / d})) r d .
$$

Finally, the hexagonal grid achieves $A / V=\sqrt{3 / 4}(1+$ $O(r / d)) r d$ which is within a factor of $\sqrt[4]{\frac{\pi^{2}}{12}} / \sqrt{3 / 4} \approx 1.0996$ of optimal. 


\section{Computing the Trap Coverage Diameter}

Even though we provide an accurate probabilistic estimate of the density needed to achieve trap coverage of a given diameter when deploying sensors randomly, it may be useful to ascertain deterministically whether a target hole diameter has been achieved after deployment, especially in the face of unanticipated and unknown deployment failures [5]. In order to determine whether a deployed network continues to provide trap coverage over time, efficient algorithms are needed to determine the largest hole diameter. In this section, we propose such algorithms.

Figure 10 shows a target region with several sensing coverage holes. Although the sensors are plotted as disks in the figure, we are not assuming a disk sensing model. Further, the sensing regions of different sensors may be different. Except in Section VI-E, where sensing regions are assumed to be star convex, the only assumptions we make are: 1) Two sensor nodes are within the transmission range of each other if their sensing regions overlap; 2) The accurate positions of nodes can be determined; 3) The boundary $\partial \mathcal{A}$ of the target region $\mathcal{A}$ is a simple polygon and is known.

To determine the largest diameter of coverage holes, the following two steps are applied. First, the boundary of each hole is found. Second, the diameters of these holes are computed based on their boundaries to obtain the largest diameter. The good news is that several ideas from existing work on discovering exact hole boundaries [6], [15], [23], [26], [29] can be applied here. However, the following challenges, which are critical to the trap coverage model, are not addressed there.

1) The boundary of a coverage hole may involve part of $\partial \mathcal{A}$, such as hole $\mathcal{H}_{7}$ in Figure 10 , so that it is hard to discover the entire boundary.

2) In a realistic sensing model, the boundary of a coverage hole may have an arbitrary shape, which makes the computation of the accurate diameter non-trivial.

3) When the shapes of sensing regions are unknown or uncertain (as in probabilistic sensing models), the boundaries of individual holes may not be accurately determined.

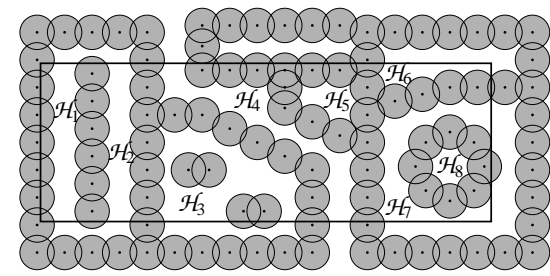

Fig. 10. An instance of deployment with eight coverage holes, $\mathcal{H}_{1}$ to $\mathcal{H}_{8}$. The rectangle shows the boundary of the target region. Note that only the holes within the target region are counted. The small disks are sensing regions.

We describe in Sections VI-B and VI-C a modification to existing algorithms that computes an accurate diameter for convex sensing regions and approximate diameter for non-convex but known sensing regions. In Section VI-E, we describe an outline of a simpler algorithm that computes an approximate diameter for both known and unknown (uncer- tain) sensing regions. We first review existing work in this area before describing our algorithms.

\section{A. Related Work}

Tools from both algebraic topology and computational geometry have been used for detecting coverage holes. Most focus on coverage verification and boundary node detection without computing the exact hole boundaries [6], [10], [15], [24], [26], [29], and several of them assume a disk sensing model and an open target region [6], [10], [24], [26], [29].

In topology based approaches, certain criteria to detect holes or verify coverage [10], [24] are derived from the topology of the covered region without using the positions of nodes. However, these criteria are computed in a centralized way and the complexity is not well studied yet. In contrast, geometry based approaches assume the positions of nodes are known [15], [26], [29] or at least the accurate distances among neighboring nodes are known [6] and use certain locally computable geometric objects to detect nodes on a coverage boundary. The first localized approach is proposed in [15] where every node can locally determine whether it is on the boundary of a $k$-coverage hole by counting the coverage levels of its sensing perimeter, which is simplified in the case of 1-coverage in [30]. The location free version of [15] is proposed in [6]. Another geometric approach uses Voronoi diagrams [9], [26], [29], which is not applicable to non-convex or heterogeneous sensing regions.

Based on [15], [23] proposes an algorithm to determine exact boundaries of coverage holes. However, it can only find those boundaries with at most one piece from $\partial \mathcal{A}$, such as $\mathcal{H}_{5}$ and $\mathcal{H}_{6}$ in Figure 10, and it assumes a disk sensing model. An algorithm to find the boundaries of routing holes is proposed in [9], and [28] proposes a method to determine the boundaries of communication holes using only the connectivity graph and a general sensing model. However, $\partial \mathcal{A}$ is not considered in either approach.

\section{B. Discovering Hole Boundary}

In this and the next section, we assume that each node knows the shape of its sensing region (not necessarily convex). The impact of sensing uncertainty is discussed in Section VI-E.

Our algorithm first applies the perimeter coverage based approach [15] to detect nodes on the boundary of coverage holes. The idea is that the sensing perimeter of one node is divided into one or more pieces by the sensing perimeters of the neighboring nodes. Every such piece is called a sensing segment, see Figure 11. According to [15], a node is on the boundary of a coverage hole iff it has a 1-covered sensing segment - a sensing segment where each point on it (except the two ends of the segment) is within the sensing region of a single node, the node where the segment belongs to.

Let $S$ denote the set of senor nodes deployed. Let $C_{S}$ denote the region covered by $S$, that is, the union of the sensing regions of the nodes in $S$, and $U_{S}$ denote the uncovered region in $\mathcal{A}$, that is, $U_{S}=\mathcal{A} \backslash C_{S}$. Recall that a coverage hole is a connected components of $U_{S}$. A connected component of $C_{S}$ 


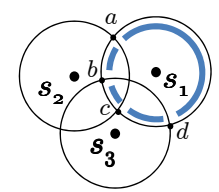

Fig. 11. $s_{2}$ and $s_{3}$ are the sensing neighbors of $s_{1}$. The four sensing segments $a b, b c, c d$, and $d a$ of $s_{1}$ are highlighted, where $d a$ is a 1-covered sensing segment.

is called a coverage component. The key idea of our solution is that all the coverage components are first discovered, which are then used along with $\partial \mathcal{A}$ to find all coverage holes.

A coverage component may or may not contain hole(s). For instance, in Figure $10, \mathcal{D}_{1}$ has two holes while $\mathcal{D}_{2}$ has no hole. The boundary of a coverage component is composed of its outermost boundary curve and the boundary of each hole in it.

Definition 6.1: Coverage cycle: A negative coverage cycle or negative cycle is the outermost boundary cycle of a coverage component. A positive coverage cycle or positive cycle is the boundary cycle of a hole in a coverage component. A coverage cycle is either a negative cycle or a positive cycle.

In Figure 10, $C_{1}, C_{3}, C_{5}, C_{6}, C_{7}, C_{11}$ are negative cycles, and the remaining ones are positive cycles. According to the observation made in [15], each coverage cycle is composed of contiguous 1-covered sensing segments.

To derive coverage holes from coverage components, we first notice that there is a partial order on the set of coverage cycles.

Definition 6.2: Coverage cycle group: For two coverage cycles $C_{i}$ and $C_{j}, C_{i} \preceq C_{j}$ if the region bounded by $C_{i}$ is included in the region bounded by $C_{j} . C_{i}$ is said to be directly enclosed in $C_{j}$ if $C_{i} \preceq C_{j}$ and there is no $k \neq j$ such that $C_{i} \preceq C_{k} \preceq C_{j}$. A coverage cycle group is a set of coverage cycles, where each group is either composed of one positive cycle and zero or more negative coverage cycles directly enclosed in the positive cycle (type 1), or composed of all the negative cycles not enclosed in any coverage cycles (type 2).

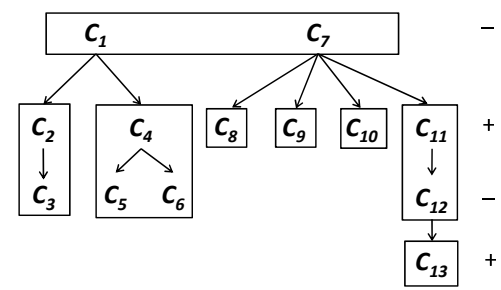

Fig. 12. The partial order among all coverage cycles in Figure 10. The cycles at the same level are either all negative(-) or all positive(+). Each box represents a coverage cycle group. $\left\{C_{1}, C_{7}\right\}$ is type 2 , and rest are type 1.

Notice that there is only one group of type 2 in any deployment. Figure 12 shows the partial order defined on the coverage cycles in Figure 10. By enumerating all the possibilities, we have the following proposition.

Proposition 6.1: Every coverage hole can be derived from a coverage cycle group and $\partial \mathcal{A}$.
Actually, there are only four possibilities as shown in Figure 10. That is, a coverage hole can be derived from (i) a positive cycle, e.g. hole $\mathcal{H}_{5}$ and $\mathcal{H}_{8}$; (ii) a positive cycle and $\partial \mathcal{A}$, e.g. hole $\mathcal{H}_{6}$; (iii) one or more negative cycles and $\partial \mathcal{A}$, e.g. hole $\mathcal{H}_{4}$; (iv) a positive cycle, one or more negative cycles, and $\partial \mathcal{A}$, e.g. hole $\mathcal{H}_{1}-\mathcal{H}_{3}$, and $\mathcal{H}_{7}$.

It follows that once all the coverage cycles are discovered and their types are determined, all the coverage holes can be derived. The boundary of coverage holes needed for diameter computation is then derived based on the following observations, which can be verified in Figure 10. First, the boundary of a coverage hole is composed of one or more cycles, but its diameter is only determined by the outermost cycle, called hole loop. For instance, the boundary of $\mathcal{H}_{3}$ in Figure 10 has two cycles, but the inner cycle - the perimeter of the two overlapped sensing regions - can be safely ignored. Second, if a hole is completely contained in another hole, it can be ignored, such as $\mathcal{H}_{8}$ in Figure 10. Third, each hole cycle is composed of sensing segments and (possibly) parts of $\partial \mathcal{A}$. If it is composed of only sensing segments, the entire cycle can be found by traversing the nodes on it. Otherwise, each piece that is composed of only sensing segments on the cycle can be found. Once all the pieces of hole boundaries are known, a polygon clipper algorithm [21] can be extended to find the hole loops by also taking $\partial \mathcal{A}$ into account.

\section{Diameter Computation}

Let $H$ denote a hole loop, and $X_{H}$ denote the set of crossings on that loop, where a crossing is defined as an intersection point of either two sensing perimeters, or a sensing perimeter with $\partial \mathcal{A}$, or a vertex of the simple polygon $\partial \mathcal{A}$. The following lemma states that $X_{H}$ is indeed a good approximation of $H$ in terms of the diameters, even if sensing region is not convex.

Lemma 6.1: $\operatorname{diam} X_{H} \leq \operatorname{diam} H \leq \operatorname{diam} X_{H}+2 D$, where $D$ is the maximum diameter of all sensing regions. Moreover, if the sensing regions are convex, then $\operatorname{diam} X_{H}=\operatorname{diam} H$.

Proof: $\operatorname{diam} X_{H} \leq \operatorname{diam} H$ follows since $X_{H} \subseteq H$. Let $x$ and $y$ be two points on $H$ with $\|x-y\|=\operatorname{diam} H$, where $\|\cdot\|$ denotes the Euclidean distance. Let $x^{\prime}$ be the crossing on $H$ closest to $x$, and $y^{\prime}$ the crossing closest to $y$. Then $\|x-y\| \leq\left\|x^{\prime}-y^{\prime}\right\|+\left\|x^{\prime}-x\right\|+\left\|y^{\prime}-y\right\| \leq\left\|x^{\prime}-y^{\prime}\right\|+2 D$. As $\left\|x^{\prime}-y^{\prime}\right\| \leq \operatorname{diam} X_{H}$, diam $H=\|x-y\| \leq \operatorname{diam} X_{H}+2 D$.

If the sensing regions are convex, then $H$ is contained within the convex hull of $X_{H}$. Since a point set and its convex hull have the same diameter, the result follows.

According to Lemma 6.1, when the sensing regions are all convex, it suffices to maintain the set of crossings on each hole loop instead of their accurate shapes in order to find the largest diameter $\mathbb{D}$. For arbitrary sensing regions, this also gives a good approximation when $\mathbb{D} \gg 2 D$.

\section{The Impact of Localization Errors}

Due to localization errors, the computed maximum hole diameter using measured node positions may be far away from the real diameter. In this section, we discuss the impact of such errors. We assume that the localization error of each node is independent and there is a maximum localization error $\Delta$ in 
terms of the Euclidean distance between the real and measured positions of a node.

Given a deployment of set of nodes $S$ in a target region $\mathcal{A}$, let $\mathbb{D}$ and $\mathbb{D}^{\prime}$ denote the real and computed largest hole diameters, respectively. Let $r_{s}$ denote the radius of node $s$, that is, the maximum distance from $s$ to its sensing perimeter. Consider the following two scenarios derived from a given deployment. In the both cases, the positions of nodes are fixed. In the first case, the sensing region of each $s \in S$ is enlarged to a similar shape with radius $r_{s}+\Delta$; in the second case, the sensing region of each $s \in S$ is shrunk to a similar shape with radius $\max \left(r_{s}-\Delta, 0\right)$. Let $\mathbb{D}_{r+\Delta}$ and $\mathbb{D}_{r-\Delta}$ denote the real hole diameters in the above two scenarios, respectively. Then, clearly $\mathbb{D}_{r+\Delta} \leq \mathbb{D}^{\prime} \leq \mathbb{D}_{r-\Delta}$. However, there is no nontrivial bound on $\left\|\mathbb{D}-\mathbb{D}^{\prime}\right\|$ for an arbitrary deployment because no matter how small $\Delta$ is, one can always construct a scenario where $\mathbb{D}=0$ and $\mathbb{D}^{\prime}=\operatorname{diam} \mathcal{A}$, or vice versa.

\section{E. Coping with Sensing Region Uncertainty}

Sensing regions show irregularity due to hardware calibration and obstacles and therefore are hard to characterize deterministically [16]. A more realistic way to characterize sensing regions is to use a sampling based approach, where the sensing region of a node is approximated by the discrete points corresponding to the events detected by this node [16]. In this section, we consider how to compute the largest diameter of coverage holes if only a limited number of samples are known. To this end, we first construct a planar graph based on the samples observed. This graph is used to approximate the real covered region, that is, the union of all the sensing regions. We then show that under certain assumptions, the largest diameter of coverage holes can by estimated by the largest diameter of the faces of this graph.

Let $B_{s}$ denote the sensing region of node $s$. We also use $s$ to denote its position and $e$ to denote the position where event $e$ happened. We make the following assumptions.

1) The positions of nodes and events observed are known.

2) Each $B_{s}$ is a star convex subset of $R^{2}$ with respect to $s$, that is, any line segment joining $s$ to a point $t$ in $B_{s}$, denoted as $\overline{s t}$, lies in $B_{s}$. Figure 13 shows an example of two overlapped star-convex sensing regions.

3) For every connected component $C_{i}$ of $B_{s_{1}} \cap B_{s_{2}}, s_{1} \neq$ $s_{2}$, there is at least one event detected in each $C_{i}$, i.e., there is a point $e_{i} \in C_{i}$ known such that $\overline{s_{1} e_{i}}$ lies in $B_{s_{1}}$ and $\overline{s_{2} e_{i}}$ lies in $B_{s_{2}}$. For instance, the two sensing regions in Figure 13 intersect at two connected subregions, with one common event detected in each subregion.

4) For each node $s$, it is known whether $B_{s}$ is completely in $\partial \mathcal{A}$, or completely outside of $\partial \mathcal{A}$, or intersects $\partial \mathcal{A}$. In the last case, the set of edges of $\partial \mathcal{A}$ that intersect $B_{s}$ is known.

Let $\mathcal{S}$ denote the set of nodes whose sensing regions are within or intersect $\partial \mathcal{A}$, and $\mathcal{E}$ denote the set of events observed by nodes in $\mathcal{S}$. Let $A$ denote the set of vertices of $\partial \mathcal{A}$. For each node $s \in \mathcal{S}$ and each edge of $\partial \mathcal{A}$ that intersects $B_{s}$, pick an arbitrary point on that edge that is within $B_{s}$, such

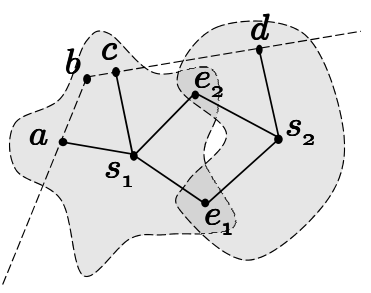

Fig. 13. The approximation of covered region by a planar graph. The dashed lines show part of $\partial \mathcal{A}$. The dashed curves show the real sensing regions (unknown) of node $s_{1}$ and $s_{2} . e_{1}$ and $e_{2}$ are two events detected by both of them. $a, c$, and $d$ are points on the edges of $\partial \mathcal{A}$ intersecting the two sensing regions, and $b$ is a vertex of $\partial \mathcal{A}$. Three faces, $\overline{a s_{1} c b a}, \overline{s_{1} e_{1} s_{2} e_{2} s_{1}}$, and $\overline{s_{1} e_{2} s_{2} d c s_{1}}$ are shown.

as points $a, c$, and $d$ in Figure 13. Name the set of such points $I$. We construct a geometric graph $G(V, E)$, where $V=$ $\mathcal{S} \cup \mathcal{E} \cup A \cup I$, and each edge in $E$ corresponds to either a line segment joining a node $s$ and an event $e$ detected by $s$, or a line segment on $\partial \mathcal{A}$ joining points in $A$ and $I$, or a line segment joining a node $s$ and a point $a$ on an edge of $\partial \mathcal{A}$ intersecting $B_{s}$. See Figure 13 for reference. Notice that, the edges of $G$ may intersect at points other than vertices. We make $G$ planar by treating these intersections as vertices as well. We then observe that $G$ is a planar graph without open faces. Let $\mathbb{D}$ and $\mathbb{D}^{\prime}$ denote the largest diameter of coverage holes and that of the faces of $G$, respectively. Then under the assumptions made above, we have the following lemma.

Lemma 6.2: $\mathbb{D} \leq \mathbb{D}^{\prime} \leq \mathbb{D}+2 D$, where $D$ is the maximum sensing diameter.

Proof: $\mathbb{D} \leq \mathbb{D}^{\prime}$ follows directly from the fact that $G$ is completely contained in the real covered region according to the second assumption made above. Consider a face of $G$, say $f$. $f$ either contains a coverage hole or is fully covered. First suppose there is a hole $\mathcal{H}$ within $f$ and let $T$ denote the set of boundary nodes on the hole loop of $\mathcal{H}$. Let $x$ and $y$ be two points on the face with $\|x-y\|=\operatorname{diam} f$. Then $x$ and $y$ must be vertices of $f$. We argue that if $x$ is not on the hole loop, then it is covered by a node in $T$. Suppose this is not true. Then there is a subgraph of $G$ induced from the nodes in $T$ and a set of common events detected by them together with part of $\partial \mathcal{A}$ that forms a polygon enclosing $\mathcal{H}$ with $x$ outside of the polygon. However, this contradicts the fact that $x$ is vertex of a face that contains the hole. Similar argument applies to $y$ as well. It follows that there exist points $x^{\prime}$ and $y^{\prime}$ on the hole loop such that $\left\|x-x^{\prime}\right\| \leq D$ and $\left\|y-y^{\prime}\right\| \leq D$. Therefore, $\|x-y\| \leq 2 D+\left\|x^{\prime}-y^{\prime}\right\| \leq 2 D+\operatorname{diam} \mathcal{H}$. That is, diam $f \leq 2 D+\operatorname{diam} \mathcal{H}$. The case where $f$ is fully covered can be viewed as the degenerate case with diam $\mathcal{H}=0$.

Notice that, the above approximation can also be applied to the case where all the sensing regions are known. It is not as accurate as the approach discussed in Section VI-B, but more efficient since the faces of $G$ and their diameters can be easily computed. If $d \gg 2 D$, the approximation may be desirable. In addition, if more events than required are detected, they can be used to improve the accuracy of the approximation. 

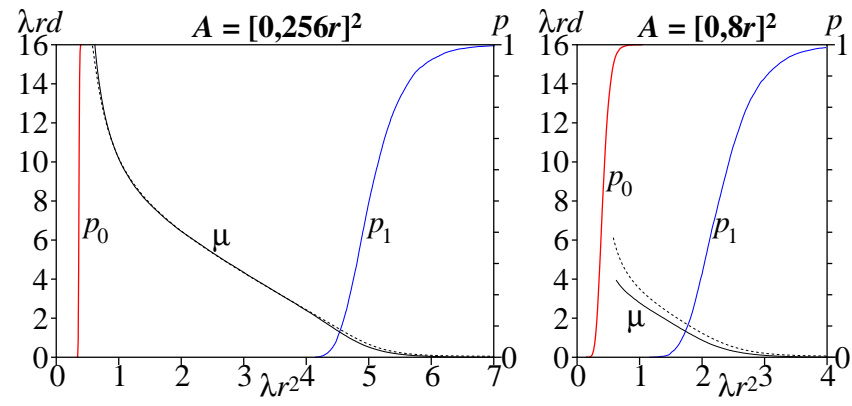

Fig. 14. Mean size of largest hole ( $\mu$, left hand scale) together with estimate based on (9) and (10) (dotted line). Probability (right hand scale) that hole size becomes finite $\left(p_{0}\right)$, i.e., percolation occurs, and probability that holes cease to exist $\left(p_{1}\right)$, i.e., full coverage occurs.

\section{F. Simulation to Validate Our Density Estimates}

In this section, we present some simulation results to support our analytical results. We consider a deployment region $\mathcal{A}$ of size $256 r \times 256 r$, where we place points according to Poisson process of intensity $N$. We vary $N$ from 0 to 500,000 and track the maximum coverage hole diameter. We repeat our experiment 10,000 times for each value of $N$ for statistical accuracy. We also ran simulations with smaller $\mathcal{A}$, obtaining very similar results even down to a $8 r \times 8 r$ region. We have two distinct goals in our simulation.

1.) Validating the accuracy of our analytical estimates.

We show results of our simulation in Figures 14 and 15. We first explain our rationale for picking the various axis before explaining the results. For $x$-axis in Figure 14, we use $\lambda r^{2}$, a dimensionless parameter which indicates the level of coverage. (Each point is covered by an average of $\pi \lambda r^{2}$ sensing regions.) We have two parameters for the $y$-axis. On the left scale, we use $\lambda r d$, a dimensionless quantity to measure the hole diameter, which also happens to be the $x$-axis in Figure 15 . Since $d$ decreases with an increase in $\lambda$ or $r$, using this unit allows us to present the entire spectrum of variation in the hole diameter in one graph. The right scale of $y$-axis in Figures 14 and the left scale in Figure 15 are probabilities. Note that the only quantity fixed in Figure 14 is the size of $\mathcal{A}$ relative to $r$.

We observe that the mean value of the maximum hole diameter observed in simulation (solid line) is mostly indistinguishable from our analytical estimates (dotted line) for $256 r \times 256 r$ region and quite close even for the $8 r \times 8 r$ region, which is smaller than many real-life deployments.

In Figure 15, we show the entire probability distribution for hole diameters for some densities, which provides significantly more information than the mean values of diameter. This confirms that our estimate of the probability distribution of hole diameters (to Poisson) and our estimation of the parameter of this distribution are highly accurate, making it quite useful in real-life deployments.

\section{2.) Graphically demonstrating the new continuum from percolation to full coverage.}

Figure 14 illustrates how the model of trap coverage fills the continuum between percolation and full coverage. The curve labeled $p_{0}$ depicts the probability of percolation, i.e., largest hole diameters becoming smaller than the deployment region. As the density increases, hole diameter decreases. The

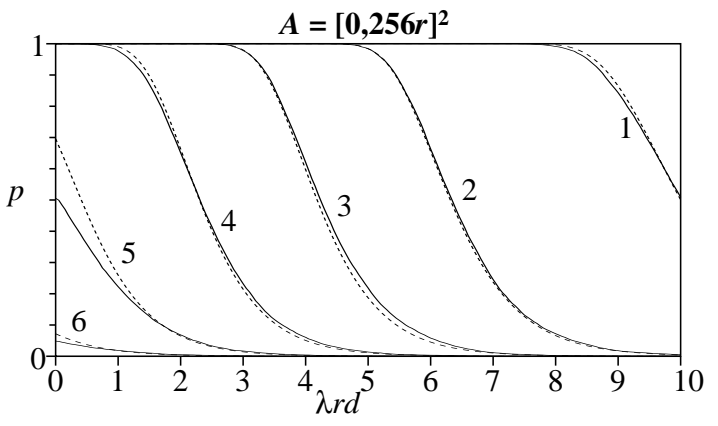

Fig. 15. Cumulative probability distribution, $\mathbb{P}\left(h_{m} \geq d\right)$, of largest hole size for $\lambda r^{2}=1, \ldots, 6$, together with estimate based on equation (9) and (10) (dotted line). For example, if $\lambda=1$, and $r=2$ (so $\lambda r^{2}=4$ ), then from Figure 14 , left side, we have $\lambda r d \approx 2$ on average (so $d \approx 1$ ), however it can range between about 1 and 6 $(d=0.5$ to 3$)$ with a probability distribution as shown here.

curve labeled $p_{1}$ depicts the probability of full coverage. As this curve approaches 1, the expected largest hole diameter approaches zero. Note that the value of $\lambda r^{2}$ corresponding to $p_{0}$ represents percolation threshold, while that corresponding to $p_{1}$ represents critical conditions for full coverage. Until this result of ours, the behavior in between these two important values of $\lambda r^{2}$ was unknown. The introduction of the trap coverage model in this paper now explains the continuum between these two important curves comprehensively, with the curve for the trap coverage diameter.

\section{CONCLUSION}

This paper generalizes the traditional model of full coverage by allowing systematic holes of bounded diameter. With this new model, deterministic guarantees on detection, particularly tracking can be maintained even if not all points in the region are covered, whether due to failure of deployed sensors or due to the expense of deploying sensors to cover every point in a large region. Trap coverage thus makes sensor deployment scalable. Of independent interest is also the fact that the trap coverage model bridges the long-standing gap between the thresholds for percolation and for full coverage.

Since Trap Coverage is a new model, several problems remain open. For example, the problem of coverage restoration upon sensor failures [18] remains open. Similarly, the problem of sleep-wakeup [7], [14], [19], [30] which has traditionally assumed full coverage model or the barrier coverage model [20], also needs to be reinvestigated for this new model. Even the problems of deployment addressed in this work needs to be reinvestigated for different sensor models (e.g., directional, mobile, etc.).

\section{ACKNOWLEDGMENT}

This work was sponsored partly by NSF Grants CNS0721983, CNS-0721817, CCF-0728928, CNS-0910878, NIH Grant U01DA023812 from National Institute for Drug Abuse (NIDA), and FIT at the University of Memphis. The content is solely the responsibility of the authors and does not necessarily represent the official views of the sponsors. 


\section{REFERENCES}

[1] R. Arratia, L. Goldstein, and L. Gordon, "Two Moments Suffice for Poisson Approximations: The Chen-Stein Method," Annals of Probability, vol. 17, pp. 9-25, 1989.

[2] X. Bai, S. Kumar, D. Xuan, Z. Yun, and T. H. Lai, "Deploying Wireless Sensors to Achieve Both Coverage and Connectivity," in ACM MobiHoc, 2006.

[3] P. Balister, B. Bollobás, A. Sarkar, and S. Kumar, "Reliable Density Estimates for Coverage and Connectivity in Thin Strips of Finite Length," in ACM MobiCom, 2007.

[4] P. Balister, Z. Zheng, S. Kumar, and P. Sinha, "Trap Coverage: Allowing Holes of Bounded Diameter in Wireless Sensor Networks," University of Memphis, CS-09-001, Tech. Rep., 2008.

[5] S. Bapat, V. Kulathumani, and A. Arora, "Analyzing the Yield of ExScal, a Large Scale Wireless Sensor Network Experiment," in IEEE ICNP, Boston, MA, 2005.

[6] Y. Bejerano, "Simple and Efficient k-Coverage Verification without Location Information," in IEEE INFOCOM, 2008.

[7] M. Cardei, M. Thai, and W. Wu, "Energy-Efficient Target Coverage in Wireless Sensor Networks," in IEEE INFOCOM, Miami, FL, 2005

[8] O. Dousse, C. Tavoularis, and P. Thiran, "Delay of intrusion detection in wireless sensor networks," in MobiHoc, 2006.

[9] Q. Fang, J. Gao, and L. J. Guibas, "Locating and Bypassing Routing Holes in Sensor Networks," in Proceedings of INFOCOM, 2004.

[10] R. Ghrist and A. Muhammad, "Coverage and Hole-detection in Sensor Networks via Homology," in IPSN, Los Angeles, California, 2005.

[11] C. Gui and P. Mohapatra, "Power Conservation and Quality of Surveillance in Target Tracking Sensor Networks," in ACM MobiCom, 2004.

[12] H. Gupta, S. Das, and Q. Gu, "Connected Sensor Cover: SelfOrganization of Sensor Networks for Efficient Query Execution," in ACM MobiHoc, 2003

[13] T. Hales, "The honeycomb conjecture," Discrete and Computational Geometry, vol. 25, no. 1, pp. 1-22, 2001.

[14] T. He and et al, "Energy-Efficient Surveillance System Using Wireless Sensor Networks," in ACM Mobisys, 2004.

[15] C. Huang and Y. Tseng, "The Coverage Problem in a Wireless Sensor Network," in ACM WSNA, 2003.

[16] J. Hwang, T. He, and Y. Kim, "Exploring in-situ sensing irregularity in wireless sensor networks," in SenSys, 2007.

[17] V. Kulathumani, M. Demirbas, A. Arora, and M. Sridharan, "Trail: A Distance Sensitive Wireless Sensor Network Service for Distributed Object Tracking," in EWSN, 2007.

[18] N. Kumar, D. Gunopulos, and V. Kalogeraki, "Sensor Network Coverage Restoration," in DCOSS, Los Angeles, CA, 2005.

[19] S. Kumar, T. H. Lai, and J. Balogh, "On $k$-Coverage in a Mostly Sleeping Sensor Network," in ACM MobiCom, 2004.

[20] S. Kumar, T. H. Lai, M. E. Posner, and P. Sinha, "Optimal Sleep-Wakeup Algorithms for Barriers of Wireless Sensors," in IEEE BROADNETS, 2007.

[21] M. Leonov, "Comparison of the different algorithms for Polygon Boolean operations," http://www.complex-a5.ru/polyboolean/comp.html.

[22] C. Sharp, S. Schaffert, A. Woo, N. Sastry, C. Karlof, S. Sastry, and D. Culler, "Design and Implementation of a Sensor Network System for Vehicle Tracking and Autonomous Interception," in EWSN, 2005.

[23] B. Tong and W. Tavanapong, "On Discovering Sensing Coverage Holes in Large-Scale Sensor Networks," Technical Report TR 06-03, Computer Science, Iowa State University, 2006.

[24] V. De Silva and R. Ghrist, "Coordinate-free Coverage in Sensor Networks with Controlled Boundaries via Homology," International Journal of Robotics Research, vol. 25, pp. 1205-1222, Dec. 2006.

[25] P. Wan and C. Yi, "Coverage by Randomly Deployed Wireless Sensor Networks," IEEE/ACM Transactions on Networking (TON), vol. 14, pp. 2658-2669, 2006.

[26] G. Wang, G. Cao, and T. L. Porta, "Movement-Assisted Sensor Deployment," in IEEE INFOCOM 2004, Hong Kong, China, 2004.

[27] X. Wang, G. Xing, Y. Zhang, C. Lu, R. Pless, and C. Gill, "Integrated Coverage and Connectivity Configuration in Wireless Sensor Networks," in ACM SenSys, 2003.

[28] Y. Wang, J. Gao, and J. S. Mitchell, "Boundary Recognition in Sensor Networks by Topological Methods," in ACM MOBICOM, 2006.

[29] C. Zhang, Y. Zhang, and Y. Fang, "Localized Algorithms for Coverage Boundary Detection in Wireless Sensor Networks," Wireless Networks, Feb. 2007
[30] H. Zhang and J. Hou, "Maintaining Sensing Coverage and Connectivity in Large Sensor Networks," in NSF International Workshop on Theoretical and Algorithmic Aspects of Sensor, Ad Hoc Wirelsss, and Peer-to-Peer Networks, 2004.

[31] — "On Deriving the Upper Bound of $\alpha$-Lifetime for Large Sensor Networks," in ACM MobiHoc, 2004. 